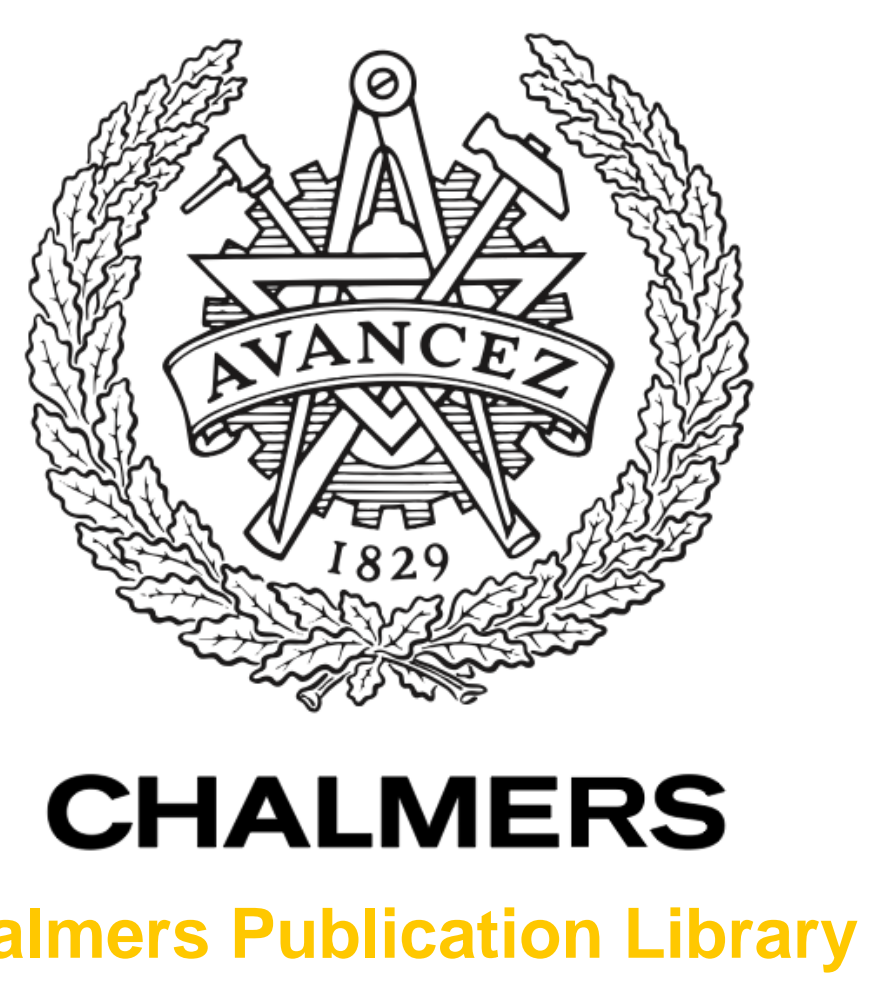

Chalmers Publication Library

Modelling of railway curve squeal including effects of wheel rotation

This document has been downloaded from Chalmers Publication Library (CPL). It is the author's version of a work that was accepted for publication in:

Notes on Numerical Fluid Mechanics and Multidisciplinary Design: Noise and Vibration Mitigation for Rail Transportation Systems - Proceedings of the 11th International Workshop on Railway Noise, Uddevalla, Sweden, 9-13 September 2013 (ISSN: 1612-2909) Citation for the published paper:

Pieringer, A. ; Baeza, L. ; Kropp, W. (2015) "Modelling of railway curve squeal including effects of wheel rotation". Notes on Numerical Fluid Mechanics and Multidisciplinary Design: Noise and Vibration Mitigation for Rail Transportation Systems - Proceedings of the 11th International Workshop on Railway Noise, Uddevalla, Sweden, 9-13 September 2013,

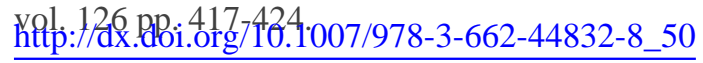

Downloaded from: http://publications.lib.chalmers.se/publication/188355

Notice: Changes introduced as a result of publishing processes such as copy-editing and formatting may not be reflected in this document. For a definitive version of this work, please refer to the published source. Please note that access to the published version might require a subscription. 


\title{
Modelling of railway curve squeal including effects of wheel rotation
}

\author{
A. Pieringer ${ }^{1}$ L. Baeza ${ }^{2}$ and W. Kropp ${ }^{1}$ \\ ${ }^{1}$ CHARMEC/Applied Acoustics, Chalmers University of Technology, \\ 41296 Göteborg, Sweden \\ Tel: +46 31772 2209, Fax: +46 31772 2212,E-mail: astrid.pieringer@chalmers.se \\ ${ }^{2}$ Centro de Investigación en Tecnología de Vehículos, Universidad Polítecnica de Valencia
}

\begin{abstract}
Summary
Railway vehicles negotiating tight curves may emit an intense high-pitch noise. The underlying mechanisms of this squeal noise are still a subject of research. Simulation models are complex since they have to consider the non-linear, transient and high-frequency interaction between wheel and rail. Often simplified models are used for wheel and rail to reduce computational effort, which involves the risk of over-simplifications. This paper focuses on the importance to include a rotating wheel instead of a stationary wheel in the simulation models. Two formulations for a rotating wheel are implemented in a previously published wheel/rail interaction model: a realistic model based on an Eulerian modal coordinate approach and a simplified model based on a rotating load and moving Green's functions. The simulation results for different friction coefficients and values of lateral creepage are compared with results obtained for the stationary wheel. Both approaches for the rotating wheel give almost identical results for the rolling speed considered. Furthermore, it can be concluded that a model of a stationary flexible wheel is sufficient for both capturing the tendency to squeal and predicting the resulting wheel/rail contact forces.
\end{abstract}

\section{Introduction}

Curve squeal is an intense high-pitch noise emitted by railway vehicles negotiating tight curves. This type of noise is commonly attributed to self-excited vibrations of the railway wheel caused by lateral sliding of the wheel on the rail [1]. The exact underlying mechanism of the curve squeal phenomenon is however still a subject of research.

Modelling curve squeal poses a challenge since the phenomenon is non-linear and transient and high-frequency wheel/rail interaction during curving is a complex process. If squeal amplitudes are of interest, time-domain models have to be formulated. Keeping the computational effort manageable necessitates simplifying the wheel/rail system, which involves the risk that over-simplified 
sub-models are used. Since curve squeal is closely linked to vibrations of the railway wheel, most squeal models include nevertheless a detailed flexible wheel model, e.g. [2-7]. However, these wheel models are generally stationary and the importance of including the effects of wheel rotation in a squeal model has - to the knowledge of the authors - so far not been investigated.

The rotation of the wheelset leads to a splitting of the resonance peaks in the frequency response of the wheel into pairs of peaks [1]. The frequency shift of the resonance peaks of the rotating wheel compared to the peaks of the stationary wheel is a result of the inertial effects due to rotation, and consequently the shift increases with the wheelset's angular velocity. Nevertheless for modes where the corresponding mode shapes produce large deflections at big distance from the wheelset axle (e.g. wheel modes in contrast to axle modes), the associated inertial effect can be important even at small velocities. In high-speed applications, the wheelset rotation has been shown to be significant for the calculation of dynamic train-track interaction, especially if the excitation takes place at frequencies where two different eigenmodes being affected by wheel rotation have coinciding resonance frequencies [8]. Wheel squeal arises essentially at low speeds during the negotiation of tight curves. Nevertheless, a possible influence of wheel rotation on squeal cannot be disregarded a priori. Squeal is known to occur predominantly at frequencies corresponding to axial modes of the wheel with zero nodal circles and several nodal diameters [1], which are affected by wheel rotation also at lower speeds. In [6], the change in wheel diameter during the lifetime of a wheel (leading to a change in the eigenfrequencies of the wheel) has been shown to significantly influence the occurrence of curve squeal. The frequency shifts due to wheel rotation are expected to be smaller than changes due to reprofiling of the wheel, but due to the strongly non-linear character of curve squeal already minor changes in parameters may lead to the sudden onset or disappearance of squeal.

The aim of the present study is two-fold. First, it will be investigated numerically with a detailed wheel/rail interaction model [7] whether it is important to include wheel rotation for the prediction of curve squeal. Results obtained with a stationary wheel will be contrasted with results obtained with a rotating wheel. Second, two different models of a rotating wheel will be compared: a realistic Eulerian approach including gyroscopic effects and centrifugal stiffening [9] and a simplified approach, where the rotating wheel is replaced by a stationary wheel with a moving load. Consequently, the study will give indications about the model complexity needed for the prediction of curve squeal.

\section{Wheel/rail interaction model}

The wheel/rail interaction model used in this study has been published earlier in [7] and is extended to take account of the effects of wheel rotation. The time-domain model includes the coupling between vertical and lateral dynamics and shows squeal in the case of a constant friction curve. Track and wheel are 
described as linear systems using pre-calculated impulse response (or Green's) functions that are derived from detailed finite element models. The time-stepping procedure is carried out by convoluting the wheel/rail contact forces with the Green's functions of wheel and track. This Green's function approach leads to high computational efficiency. Measured wheel and rail profiles are used and the wheel/rail contact position has been pre-calculated for an assumed lateral displacement of the wheel on the rail [7]. Wheel and rail model have been chosen based on the conditions in a curve in the network of Stockholm metro, where severe corrugation and squeal occur [10].

\subsection{Wheel model}

The wheel considered is a C20 metro wheel (radius $0.39 \mathrm{~m}$, mass $213 \mathrm{~kg}$ ), which is clamped at the centre of the hub. It has been modelled with three-dimensional 20-node solid elements with a commercial finite-element (FE) software. The model contains a total of 7920 elements and 37800 nodes. For the stationary wheel, the wheel is represented by the vertical point receptance, the lateral point receptance and the vertical/lateral cross receptance calculated with the FE-model at the pre-determined contact point on the wheel tread. The eigenmodes of the wheel up to $7 \mathrm{kHz}$ have been included in the receptances. The Green's functions corresponding to the receptances are obtained by inverse Fourier transform. The inclusion of the wheel rotation is described in Section 3.

\section{$2.2 \quad$ Track model}

The track model consists of one continuously supported rail [7] of type BV50, which is a common Swedish rail type. It is built with waveguide finite elements using the software package WANDS [11]. In the interaction model, the track is represented by moving Green's functions, which include the motion of the contact point along the rail [7, 12]. For simplicity, the lateral motion of the contact point has been neglected. The moving Green's functions give the displacement response of the rail due to an impulse excitation of the rail at time $t=0$ and position $x=x_{0}$ at a point moving along the rail with train speed $v$ away from the excitation point. The discrete version of the moving Green's functions is constructed from ordinary Green's functions obtained from a series of track transfer receptances calculated with the waveguide finite element model of the rail. This procedure requires that space and time resolution are linked by the train speed: $\Delta x=v \Delta t$. The dynamics of the track in vertical direction, in lateral direction and the coupling between the vertical and lateral dynamics have been included.

\subsection{Contact model}

The contact model used is an implementation of Kalker's model CONTACT [13], which is a three-dimensional, non-steady state rolling contact model based on the assumption that wheel and rail can be locally approximated by elastic half-spaces. In addition to the parameters included in CONTACT, the contact model used in this study considers the contribution of the structural dynamics of wheel and track to the creepages. The contact model is solved online in the time-stepping procedure. 


\section{Inclusion of wheel rotation}

Two different models of a rotating wheel are considered: a realistic Eulerian approach and a simplified approach.

\subsection{Realistic approach of a rotating wheel}

The realistic model of the rotating wheel is based on an Eulerian modal coordinate approach [9] which uses the finite element methodology for obtaining the equation of motion terms. The model adopts a modal approach where mode shapes are used as basis functions in the displacement formulation. The model takes advantage of the solid of revolution geometry since the mode shapes are implemented in non-rotating coordinates. The Eulerian approach avoids the need to introduce a rotating frame of reference, but the kinematics of the material particles of the solid are not explicit: the coordinate system is associated with the displacement of a particle that at instant $t$ occupies an arbitrary spatial position. This coordinate system is more efficient because the forces in the wheel-rail contact and the primary suspension are referred to in a non-rotating frame. The resultant mathematical model is linear and its equation of motion matrices are constant and can be pre-calculated. Based on the mathematical model, receptances are calculated at the pre-determined contact point on the wheel tread. In the interaction model, the rotating wheel is represented by the corresponding (ordinary) Green's functions.

\subsection{Stationary wheel with moving load}

The simplified approach is based on a stationary wheel with a time-varying load rotating around its circumference with rolling speed. In comparison to the Eulerian modal coordinate approach, this simplified approach neglects the inertial effects associated with the rotation (gyroscopic effect, stiffening and radial extension of the structure due to centrifugal forces) and it only considers the moving load effect. However, this assumption produces similar results to the ones from the more realistic model, see Section 3.3. In the interaction model, the stationary wheel with moving load is represented by moving Green's functions $[7,12]$ that incorporate the rotation of the wheel. These functions contain the displacement value at a point rotating around the wheel circumference with rolling speed due to an impulse excitation at $t=0$ at angular position $\phi=\phi_{0}$ of the stationary wheel. The moving Green's functions are constructed by selecting the displacement value for each time step at the corresponding contact point from pre-calculated ordinary Green's functions for all potential contact points around the wheel circumference. In the same manner as for the rail, this procedure requires the time and space coordinates being linked by the rolling speed.

\subsection{Comparison of the wheel models}

As an example, Figure 1 shows a comparison of the lateral wheel receptances in the Eulerian coordinate system (i.e. fixed to the contact point between wheel and rail) obtained for a train speed of $v=50 \mathrm{~km} / \mathrm{h}$ for the two approaches of the rotating 
wheel and the stationary wheel. Both approaches for the rotating wheel show almost identical response functions revealing a frequency split due to the rotation of the wheel. There are only very small deviations due the influence of the additional effects considered in the Eulerian modal coordinate approach such as centrifugal stiffening and the gyroscopic effect. The small additional peaks visible in the receptance obtained for the stationary wheel with moving load are due to numerical problems with the spatial interpolation. The FE mesh (which was kept identical for all three approaches) only provided a circumferential resolution of $3.4 \mathrm{~cm}$ while a resolution in the order of millimetres is needed for the construction of the moving Green's functions. Different interpolation approaches led to different small additional peaks. These minor effects, which could be avoided by modelling the wheel with axi-symmetric finite elements [7], should however not influence the simulation results from the squeal model.

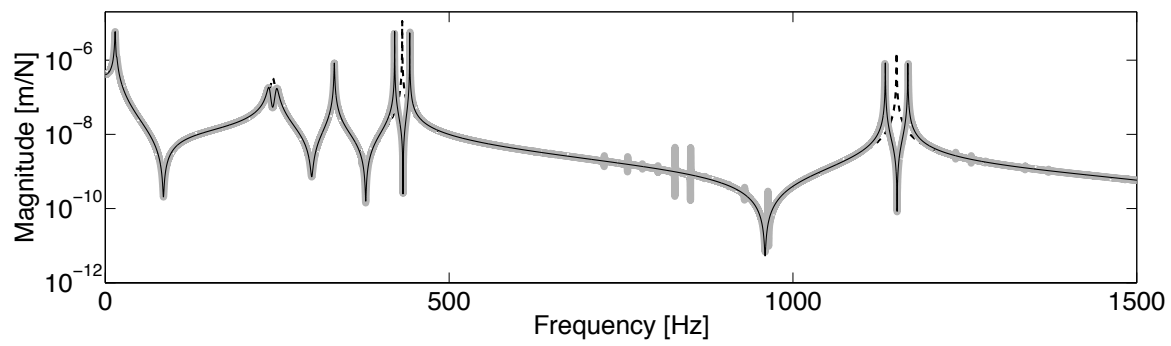

Fig. 1. Magnitude of the lateral wheel receptance for train speed $v=50 \mathrm{~km} / \mathrm{h}$ : stationary wheel (---), stationary wheel with moving load (_- grey), rotating wheel (-).

\section{Simulation results}

Applying the three different sets of pre-calculated Green's functions for the wheel, the dynamic wheel/rail interaction during curving has been calculated for a range of input parameters. For a train speed of $v=50 \mathrm{~km} / \mathrm{h}$, combinations of different values of lateral creepage and friction coefficient were investigated. Similar as in [7], the resulting lateral contact force $F_{2}$ was expressed as a force level according to

$$
L_{F_{2}}=20 \log \frac{F_{2, \text { rms }}}{1 \mathrm{~N}} .
$$

The rms-value $F_{2 \text {,rms }}$ of the transient part of the signal was obtained as

$$
F_{2, \text { rms }}=\sqrt{\frac{1}{T} \int_{t_{1}}^{t_{1}+T}\left(F_{2}(t)-\bar{F}_{2}\right)^{2} \mathrm{~d} t},
$$

where $\bar{F}_{2}$ is the mean value of the force in the considered time interval. The rms-value was calculated from the last $0.15 \mathrm{~s}$ of the force signal, which in total was $3.5 \mathrm{~s}$ long. In cases where stick/slip oscillations (corresponding to curve squeal) develop, the transient part of the force signal is large and leads to a high force level $L_{F 2}$. 
Figure 2 shows the results of the simulations for the stationary wheel. In five of the eight investigated cases, curve squeal occurred. Figure 3 presents the results for the same simulations, when including a rotating wheel. The results are represented as differences in force level with regard to the stationary wheel. The differences to the stationary wheel results and the differences between the two models including wheel rotation are small in cases where squeal occurs. Where no squeal occurs (i.e. for low force levels in Figure 2) high differences can be seen, especially for the stationary wheel with a moving load. However, since the signal itself (i.e. the transient part of the lateral force) is vanishingly small in these cases, the differences are not considered relevant.

In the cases where squeal occurs not only is the energy content in the lateral forces almost identical for the three wheel models, but so too are the time behaviour and the spectra of the steady-state stick-slip motion as exemplified in Figure 4 for one of the cases. The only major difference occurs in the transient build-up process. In the simulations including a rotating wheel more time is needed to build up the stationary slip-stick motion.

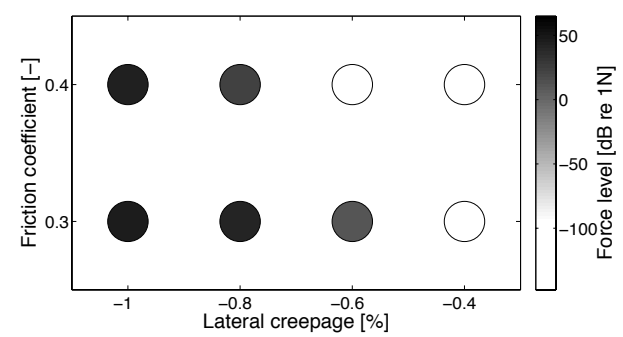

Fig. 2. Simulation results for the stationary wheel: force level calculated according to Equation (4.1).

a)

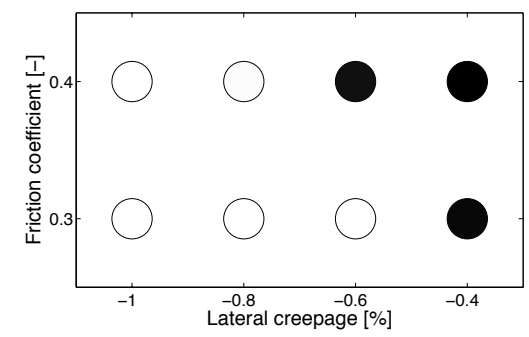

b)

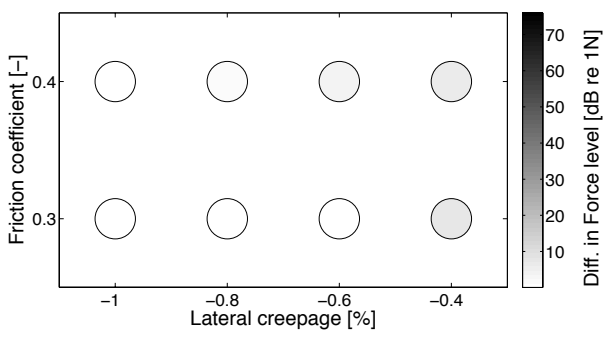

Fig. 3 Simulation results for the stationary wheel with moving force (a) and the rotating wheel(b): difference in force level with regard to the stationary wheel $\left(\mathrm{L}_{\mathrm{F} 2}-\mathrm{L}_{\mathrm{F} 2 \text {,stat }}\right)$.

\section{Conclusions}

Three different models for the wheel were included in an existing model for the simulation of curve squeal: a model of a stationary wheel, a simplified approach for a rotating wheel including only the moving load effect and a realistic approach 
a)

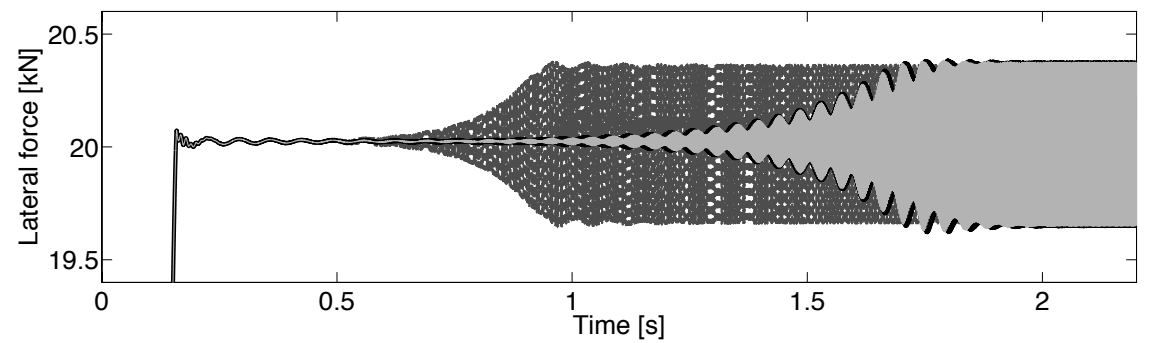

b)

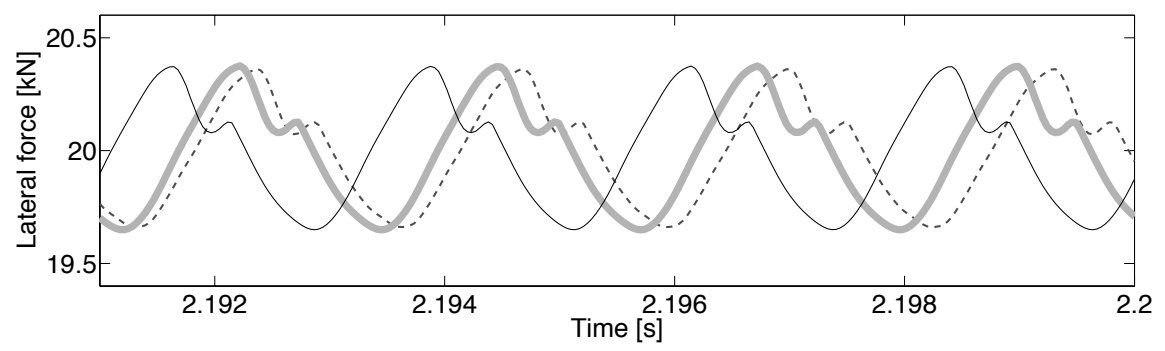

c)

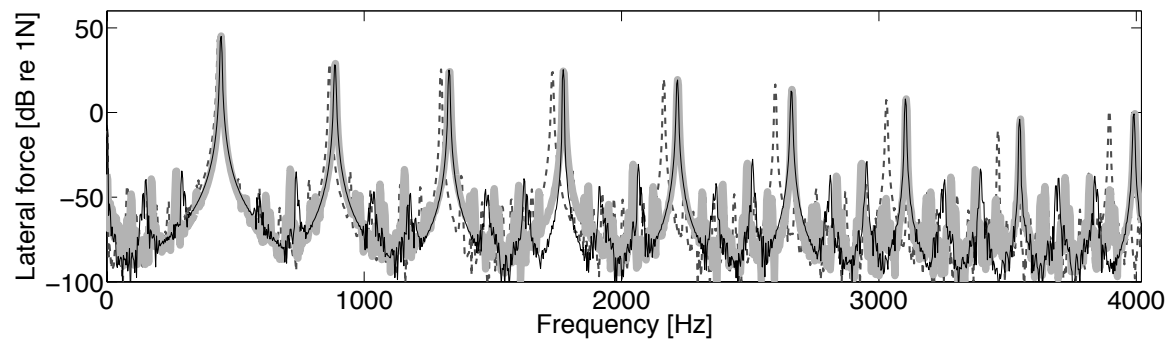

Fig. 4. Lateral contact force for the case $\mu=0.3$ and $\eta=-1 \%$; stationary wheel (----, dark grey), stationary wheel with moving load (- light grey), rotating wheel (-); a) time series, b) zoom on the time series, c) power spectrum.

for a rotating wheel considering centrifugal stiffening and gyroscopic effects. The wheel receptances obtained from both rotating wheel models were almost identical. In the dynamic simulations, all three wheel models gave similar results for different combinations of friction coefficient and lateral creepage in terms of the lateral contact forces. On the one hand, these results indicate that the stationary wheel with moving load is a valid approach to include the wheel rotation, at least for the train speed of $50 \mathrm{~km} / \mathrm{h}$ considered in this study. On the other hand, it can be concluded that using a stationary wheel is sufficient for both capturing the tendency to squeal and predicting the resulting lateral forces. Relevant differences occurred only during the build-up of the stick/slip oscillation. The rotation of the wheel delays the build-up process, but it does not reduce the final force amplitudes. 
However, the vibration pattern on the wheel will change due to rotation. This is expected to influence the radiation from the wheel. Therefore, the influence of wheel rotation on sound radiation needs to be investigated further.

\section{Acknowledgements}

The first author acknowledges the financial contribution of the Chalmers Area of Advance "Transport". The second author acknowledges the financial support through the project TRA2010-15669 of the Spanish Ministry of Economy and Competitiveness.

\section{References}

[1] Thompson, D.: Railway noise and vibration: Mechanisms, modelling and means of control, Elsevier, Oxford, UK, 2009.

[2] Fingberg, U.: A model for wheel-rail squealing noise, J. Sound Vib., 365-377 (1990).

[3] Périard, F.J.: Wheel-rail noise generation: Curve Squealing by Trams. PhD thesis, Technische Universiteit Delft, 1998.

[4] Chiello, O., Ayasse, J.-B., Vincent, N., Koch, J.-R.: Curve squeal of urban rolling stock - Part 3: Theoretical model, J. Sound Vib., 293, 710-727 (2006).

[5] Brunel, J.F., Dufrénoy, P, Naït, M., Muñoz, J.L., Demilly, F: Transient model for curve squeal noise. J. Sound Vib., 293, 758-765 (2006).

[6] Glocker, Ch., Cataldi-Spinola, E., Leine, R.I.: Curve squealing of trains: Measurement, modelling and simulation. J. Sound Vib., 324, 365-386 (2009).

[7] Pieringer, A.: Time-domain modelling of high-frequency wheel/rail interaction, PhD thesis, Chalmers University, Göteborg, Sweden, 2011.

[8] Torstensson, P.T., Nielsen, J.C.O., Baeza, L.: Dynamic train-track interaction at high vehicle speeds - Modelling of wheelset dynamics and wheel rotation, J. Sound Vib., 330, 5309-5321 (2011).

[9] Martínez-Casas, J., Mazzola, L., Baeza, L., Bruni, S.: Numerical estimation of stresses in railway axles using train-track interaction model, Int. J. Fatigue, 47, 18-30 (2013).

[10] Torstensson, P.T., Nielsen, J.C.O.: Monitoring of rail corrugation growth due to irregular wear on a railway metro curve, Wear, 267, 556-561 (2009).

[11] Nilsson C.-M., Jones, C.J.C., Thompson, D.J., Ryue, J.: A waveguide finite element and boundary element approach to calculating the sound radiated by railway and tram rails, J. Sound Vib., 321, 813-836 (2009).

[12] Nordborg, A.: Wheel/rail noise generation due to nonlinear effects and parametric excitation, J. Acoust. Soc. Am., 111(4), 1772-1781 (2002).

[13] Kalker, J.J.: Three-dimensional elastic bodies in rolling contact. Kluwer Academic Publishers, Dordrecht, Boston, London, 1990. 